\title{
WFirst Ultra-Precise Astrometry II: Asteroseismology
}

\author{
Andrew Gould ${ }^{1}$, Daniel Huber ${ }^{2,3,4}$, Matthew Penny ${ }^{1,5}$, And Dennis Stello ${ }^{4,6}$ \\ ${ }^{1}$ Department of Astronomy, Ohio State University, 140 W. 18th Ave., Columbus, OH 43210, USA \\ gould@astronomy.ohio-state.edu \\ ${ }^{2}$ NASA Ames Research Center, Moffett Field, CA 94035, USA \\ ${ }^{3}$ SETI Institute, 189 Bernardo Avenue, Mountain View, CA 94043, USA \\ ${ }^{4}$ Sydney Institute for Astronomy (SIfA), School of Physics, University of Sydney, NSW 2006, Australia \\ huber, stello@physics.usyd.edu.au \\ ${ }^{5}$ Sagan Fellow \\ ${ }^{6}$ Stellar Astrophysics Centre, Department of Physics and Astronomy, Aarhus University, Ny Munkegade 120, DK-8000 \\ Aarhus C, Denmark
}

Received October 28, 2014; accepted December 30, 2014

\begin{abstract}
WFIRST microlensing observations will return high-precision parallaxes, $\sigma(\pi) \lesssim 0.3 \mu$ as, for the roughly 1 million stars with $H<14$ in its $2.8 \mathrm{deg}^{2}$ field toward the Galactic bulge. Combined with its 40,000 epochs of high precision photometry $\left(\sim 0.7\right.$ mmag at $H_{\text {vega }}=14$ and $\sim 0.1 \mathrm{mmag}$ at $\left.H=8\right)$, this will yield a wealth of asteroseismic data of giant stars, primarily in the Galactic bulge but including a substantial fraction of disk stars at all Galactocentric radii interior to the Sun. For brighter stars, the astrometric data will yield an external check on the radii derived from the two asteroseismic parameters, the large-frequency separation $\left\langle\Delta \nu_{n l}\right\rangle$ and the frequency of maximum oscillation power $\nu_{\max }$, while for the fainter ones, it will enable a mass measurement from the single measurable asteroseismic parameter $\nu_{\max }$. Simulations based on Kepler data indicate that WFIRST will be capable of detecting oscillations in stars from slightly less luminous than the red clump to the tip of the red giant branch, yielding roughly 1 million detections.
\end{abstract}

Key words: astrometry — gravitational microlensing — stars: oscillations

\section{INTRODUCTION}

Kepler's wide-field, high-precision photometry has revolutionized asteroseismology. Its $\mu$ mag precision at 30 minute cadence for brighter targets has enabled asteroseismic measurements for more than 15,000 giant stars, while its special high (1 minute) cadence feature has even permitted measurements for more than 500 dwarfs. Kepler has built on the earlier successes of CoRoT, which observed a smaller overall number of stars but had the added advantage of probing several different lines of sight. See Chaplin \& Miglio (2013) for a review.

As a major part of its overall mission, the WFIRST satellite will conduct a photometric survey of $2.8 \mathrm{deg}^{2}$ field toward the Galactic bulge, with roughly 40,000 52s exposures in a broad $H$ band during six 72-day campaigns at 15 minutes cadence using its $2.4 \mathrm{~m}$ telescope. While the main purpose of these campaigns is a search for extrasolar planets using the gravitational microlensing technique, the survey will produce a wealth of astrometric and photometric data that can be applied to a wide range of other astronomical questions. In Gould (2014a) (hereafter Paper I), we showed how this astrometric data set could be used to obtain precise orbits of several thousand Kuiper Belt Objects (KBOs) down to $H_{\text {vega }} \sim 28.2$ (corresponding to $R \sim 29.6$ ) and how the photometric data could measure thousands of KBO

CORRESPONDING AUthor: A. Gould occultations. These measurements will be concentrated at one extreme of WFIRST sensitivity, well below the effective "sky" (actually a combination of true sky, read noise, and dark current) of 341 photons per pixel per exposure.

Here we consider an application from the opposite extreme, astrometry and photometry of "saturated" stars, i.e., stars that fill up the full well of the central pixel even in the first 2.6s "read" of the 52s exposure. Because a diffraction-limited point spread function (PSF) for a circular aperture falls off roughly as $r^{-3}$, these "saturated" stars still produce excellent astrometry and photometry. Indeed, the mission-length parallax precision, $\sigma(\pi) \lesssim 0.3 \mu$ as for about 1 million stars, is likely to vastly surpass the performance of any other instrument.

By contrast, the photometric precision, roughly $\sigma(H) \sim 10^{(2 / 15)(H-15)} \mathrm{mmag}$ for stars of $8 \lesssim H \lesssim 15$ is substantially worse than Kepler's, and this for primarily the same reason that its astrometry is better: whereas Kepler deliberately degraded its PSF to spread brightstar photons over many pixels (Table 1 of Koch et al. 2010), WFIRST will operate near the $2.4 \mathrm{~m}$ diffraction limit, thus "squandering" most of the photons from the brightest stars. In addition, WFIRST will spend only $10 \%$ of its time observing each of 10 subfields, but this is basically compensated by its larger mirror relative to Kepler.

WFIRST suffers a second asteroseismic disadvantage relative to Kepler, in addition to worse photometric pre- 
cision: it operates in a broad $H$ band rather than $K e$ pler's broad visible band. Because the amplitudes of stellar oscillations are only about $45 \%$ as big at $H$ band, this is equivalent to a factor $\sim 2$ further degradation in precision.

However, WFIRST partially compensates for these disadvantages with its high-precision astrometry. To first order, asteroseismic measurements of cool stars yield two parameters, the large-frequency separation $\left\langle\Delta \nu_{n l}\right\rangle$ and the frequency of maximum oscillation power $\nu_{\max }$. The first is a measure of mean density $\rho$ (Ulrich 1986; Kjeldsen \& Bedding 1995),

$$
\frac{\rho}{\rho_{\odot}} \simeq\left(\frac{\left\langle\Delta \nu_{n l}\right\rangle}{\left\langle\Delta \nu_{n l}\right\rangle_{\odot}}\right)^{2}
$$

while the second is a measure of the surface gravity $g$ (Brown et al. 1991; Kjeldsen \& Bedding 1995),

$$
\frac{g}{g_{\odot}} \simeq \frac{\nu_{\max }}{\nu_{\max , \odot}}\left(\frac{T_{\mathrm{eff}}}{T_{\mathrm{eff}, \odot}}\right)^{1 / 2},
$$

where $T_{\text {eff }}$ is the effective temperature.

Obviously, these can be combined to yield the star's radius and mass (Kallinger et al. 2010),

$$
\frac{R}{R_{\odot}} \simeq \frac{\nu_{\max }}{\nu_{\max , \odot}}\left(\frac{\left\langle\Delta \nu_{n l}\right\rangle}{\left\langle\Delta \nu_{n l}\right\rangle}\right)^{-2}\left(\frac{T_{\mathrm{eff}}}{T_{\mathrm{eff}, \odot}}\right)^{1 / 2},
$$

and

$$
\frac{M}{M_{\odot}} \simeq\left(\frac{\nu_{\max }}{\nu_{\max , \odot}}\right)^{3}\left(\frac{\left\langle\Delta \nu_{n l}\right\rangle}{\left\langle\Delta \nu_{n l}\right\rangle_{\odot}}\right)^{-4}\left(\frac{T_{\mathrm{eff}}}{T_{\mathrm{eff}, \odot}}\right)^{3 / 2} .
$$

These "equations" (really scaling relations) point to four related problems in the interpretation of asteroseismic measurements. First, the relations are approximate and depend as well on evolutionary stage and chemistry (Stello et al. 2009; White et al. 2011; Miglio et al. 2013). The only way to verify that real masses are being extracted from Equation (4) is to apply it to rare test cases of stars with masses that are measured from binary-star orbits ${ }^{\mathbf{1}}$ (Sandquist et al. 2013; Frandsen et al. 2013), or to apply the companion Equation (3) to stars with known radii (Huber et al. 2012; White et al. 2013). In the Gaia era, there will be a large number of giant stars in the solar neighborhood (within 1 $\mathrm{kpc})$ with accurate $(\lesssim 1 \%)$ parallaxes, and so accurate radii based on the well measured infrared color/surfacebrightness relations. This includes several hundred giants with Kepler asteroseismology, which will therefore provide crucial checks on these scaling relations, as well as calibrations of the deviations from them based on various other observables. However, it will not be easy to extend these calibrations based on "garden variety" solar-neighborhood stars to the much more extreme populations found in the Galactic bulge.

\footnotetext{
${ }^{1}$ See Epstein et al. (2014) for a less stringent but still interesting such test.
}

Second, errors in the observed quantities $\nu_{\max }$ and $\left\langle\Delta \nu_{n l}\right\rangle$ are amplified by factors of three and four respectively before they enter the mass. Thus even if the deviations from the scaling relations are understood perfectly, it can be difficult to extract masses (hence ages) of individual stars.

Third, the assumption underlying the $\nu_{\max }$ scaling relation proposed by Brown et al. (1991) (a linear relation of $\nu_{\max }$ with the acoustic cut-off frequency) is theoretically less understood than the scaling of $\left\langle\Delta \nu_{n l}\right\rangle$, potentially introducing unknown systematic errors in mass estimates based on Equation (4). While theoretical work explaining the $\nu_{\max }$ relations has yielded some progress (e.g., Belkacem et al. 2011), mass measurements based on $\left\langle\Delta \nu_{n l}\right\rangle$ should therefore yield results that we can more comfortably interpret.

Fourth, if the data are sufficiently noisy, it will be possible to measure only $\nu_{\max }$ (and not $\left\langle\Delta \nu_{n l}\right\rangle$ ), which would yield only a surface gravity but not a mass.

All four of these problems can be solved or mitigated by WFIRST parallaxes, which yield stellar radii (assuming the surface brightness can be properly estimated). First, for brighter stars, which have both better photometric precision and larger amplitudes, it will be possible to directly check the radius derived by applying Equation (3) to asteroseismic measurements. Second, if the radius $R$ is known independently, then the mass can be written

$$
\begin{gathered}
\frac{M}{M_{\odot}} \simeq\left(\frac{\left\langle\nu_{n l}\right\rangle}{\left\langle\nu_{n l}\right\rangle}\right)^{2}\left(\frac{R}{R_{\odot}}\right)^{3}, \\
\frac{M}{M_{\odot}} \simeq \frac{\nu_{\max }}{\nu_{\max , \odot}}\left(\frac{T_{\mathrm{eff}}}{T_{\mathrm{eff}, \odot}}\right)^{1 / 2}\left(\frac{R}{R_{\odot}}\right)^{2} .
\end{gathered}
$$

For higher $\mathrm{S} / \mathrm{N}$ detections, Equation (5) removes the dependency of the mass estimate on the less well-tested $\nu_{\max }$ scaling relation (and so permits precise testing of this relation), while for low $\mathrm{S} / \mathrm{N}$ detections, Equation (6) still permits a mass measurement (whose accuracy will be determined by the tests just mentioned).

Hence, WFIRST astrometry can transform relatively crude WFIRST asteroseismic measurements into precision mass and (when metallicities are available) age measurements.

\section{Astrometric and Photometric Precision Saturated Stars in the Photon-Noise Limit}

The first of many steps toward estimating the precision of parallaxes and asteroseismic parameters is to establish the precision of individual measurements in the photon-noise limit. Of course, since the parallax precisions of $0.3 \mu$ as claimed above are $10^{5.6}$ smaller than the $W F I R S T$ pixel size, it is clear at the outset that even very subtle systematics may be the ultimate limit of these measurements. Nevertheless, a clear understanding of the statistical errors is an essential prerequisite for a discussion of systematics.

Figure 1 shows the results of a Fischer matrix calculation of the astrometric and photometric errors. We 

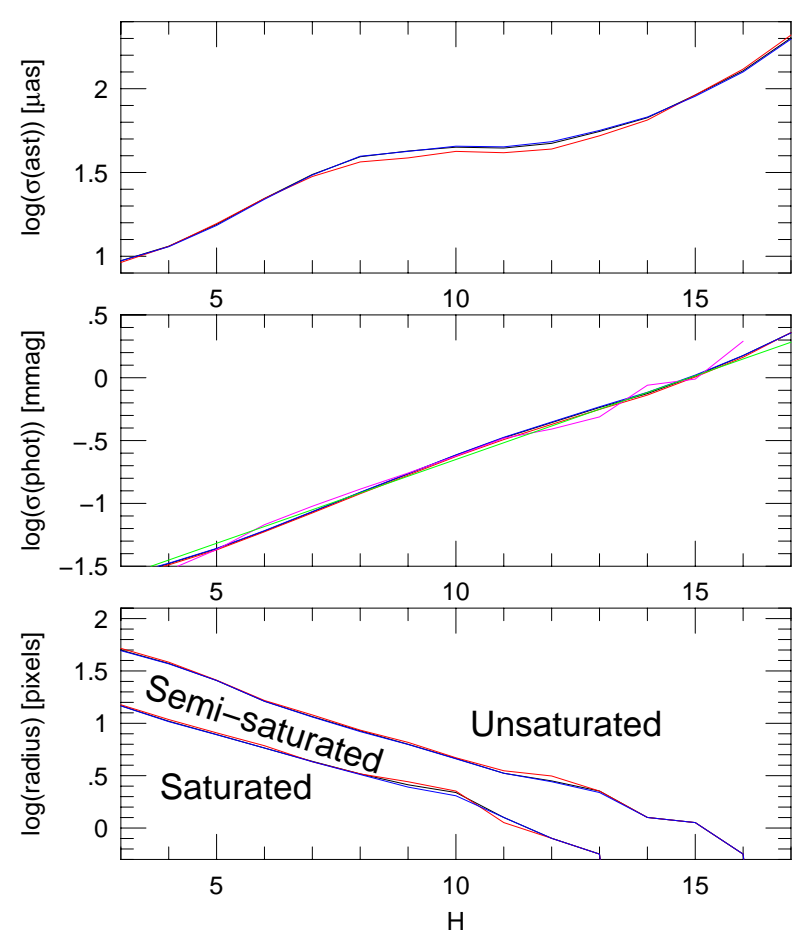

Figure 1. Astrometric (top) and photometric (middle) precision from a single-epoch of simulated WFIRST observations as a function of $H$-band apparent magnitude. Red, black and blue are for thermal spectral energy distributions at 2500,4500 , and $6500 \mathrm{~K}$. In the middle panel, the magenta curve shows the analytic calculation (Equation (16)) using $r_{\text {unsat }}$, calculated numerically from $\pi r_{\text {unsat }}^{2}=N_{\text {sat }}+$ $N_{\text {semi-sat }}$ (total number of saturated and semi-saturated pixels) as displayed in the bottom panel (upper curves). The agreement is excellent. Also shown in green is a pure power law $\log \sigma$ (phot) $=(2 / 15) H+$ const. The approximately flat astrometric errors $\sigma$ (ast) $\sim 60 \mu$ as (top panel) are predicted for an Airy disk when $r_{\text {sat }}>0$ (bottom panel). However, this relation holds only for $H \gtrsim 8$, brighter than which the diffraction spikes start to contribute to the precision.

begin with a $2048 \times 2048$ representation of the PSF for monochromatic $1.0 \mu \mathrm{m}$ light, with 11 mas pixels, i.e., 10 times smaller than the WFIRST pixels. We then convolve this with several different spectral energy distributions, with the PSF scaled by wavelength. We find numerically that the results do not vary significantly for radically different spectral energy distributions. We assume (as in Paper I) 52s exposures, a photometric zero point of $H_{\mathrm{vega}}=26.1$, and 341 photo-electrons per pixel in sky, read noise, and dark current. We assume that the full well has a depth of 100,000 photo-electrons, and that it is read in 20 equal 2.6s "reads". Hence, for example, if $52 \mathrm{~s}$ of flux would generate 2,000,001 photoelectrons, the pixel would be saturated; for 1,999,980 electrons a single read would record 99,999 , so the fractional photon-noise error would be $0.32 \%$; for $1,000,020$ electrons, a single read would record 50,001, so the fractional error would be $0.45 \%$, etc. In spite of this somewhat irregular pattern, the basic effect of this read structure is that the fractional errors are near $0.32 \%$ over the entire range $1 \times 10^{5}<N<2 \times 10^{6}$. At higher fluxes there is no information while at lower fluxes the errors increase as $1 / \sqrt{N}$ in the usual way.

We explicitly assume two things that are known not to be true. First, we assume that the sensitivity of the pixel is spatially uniform. Second, we assume that the detector response is linear below saturation. These assumptions have no practical impact on the signal-tonoise calculations, although they will have to be carefully taken into account in the actual measurements.

The astrometric covariance matrix is then given by

$$
c=b^{-1}, \quad b_{i, j}=(\delta \theta)^{-2} \sum_{k} \frac{\left(\delta \ln F_{k, i}\right)\left(\delta \ln F_{k, j}\right)}{\left[\sigma\left(\ln F_{k}\right)\right]^{2}}
$$

where $\delta \ln F_{k, i}$ is the fractional flux change when pixel $k$ is displaced in the $i$ (either $x$ or $y$ ) direction by an angle $\delta \theta$ (in practice 0.1 pixels, i.e., 11 mas), and $\sigma\left(\ln F_{k}\right)$ is the fractional photon error, discussed immediately above. The fractional photometric error is simply $\left[\sum_{k} \sigma\left(\ln F_{k}\right)^{-2}\right]^{-1 / 2}$.

When discussing systematics, it will be important to understand the origin of the principal features of Figure 1. The photometric error is an essentially featureless power law, $\sigma(\ln F) \propto F^{-1 / 3}$, which appears as $\log \sigma(\ln F)=(0.4 / 3)(H-15) \sim 0.13(H-15)$ on the plot. By contrast, the astrometric error is essentially flat over $8<H<14$, which is the principal range of interest for the current work. At both fainter and brighter magnitudes, it approaches slightly different power-law slopes of $-1 / 2$ and $-1 / 3$, respectively.

The WFIRST PSF is basically comprised of two components: an Airy disk and 12 diffraction spikes. The main features of Figure 1 can be understood analytically from these two components.

\subsection{Astrometric Precision of Saturated Broad Band Airy Disk}

The diffraction pattern due to a circular mirror of diameter $D$ for monochromatic light of wavelength $\lambda$ is an "Airy disk",

$$
I=4 I_{0}\left(\frac{J_{1}(x)}{x}\right)^{2} ; x=\pi \frac{D}{\lambda} \sin \theta ; I_{0}=P_{0}\left(\frac{\pi D}{2 \lambda}\right)^{2}
$$

where $I$ and $I_{0}$ are intensity in power per steradian, $J_{1}$ is a Bessel function, and $P$ the total power incident on the mirror. The asymptotic behavior of the Bessel function is $J_{1}(x) \rightarrow(\pi x / 2)^{-1 / 2} \cos (x-3 \pi / 4)$. Hence, the Airy disk scales $I \sim \cos ^{2}(x-3 \pi / 4) / x^{3}$.

We now focus on applications to the very broad $W F I R S T$ wide $H$-band filter, which covers a factor $\sim 2$ in wavelength. The fourth zero $(x=13.324=4.21 \pi)$ of the central wavelength $\left(\lambda_{0}=1.5 \mu \mathrm{m}\right)$ at $\theta=542 \mathrm{mas}$, corresponds a range of $6.36>x / \pi>3.18$ for wavelengths $(2 / 3) \lambda_{0}<\lambda<(4 / 3) \lambda_{0}$. Hence, even at a few Airy lengths, the combined flux per pixel from all wavelengths is well approximated by

$$
F(r)=\frac{k}{r^{\gamma}} ; \quad \gamma=3
$$


where $r$ indicates the distance of the pixel from the center in pixels and $k$ is a constant. Of course, there will be some residual wavy structure superposed on this power law, and this will have to be taken into account in the actual measurement. However, from the standpoint of determining the astrometric precision this is unimportant. Moreover, to the extent that this structure enters, it adds information, and therefore ignoring it is conservative. More generally, we can consider an arbitrary power law $r^{-\gamma}$.

Let us now consider that the well has a capacity for $n_{\max }=10^{5}$ photo-electrons and that there are $N_{\text {read }}=20$ reads during the exposure, so that pixels with more than $n_{\text {max }} N_{\text {read }}=2 \times 10^{6}$ photo-electrons are fully saturated (no information). We define $r_{1}$ as the radius at which $n_{\max }$ photo-electrons are captured in a single read time, and $r_{m}=m^{1 / \gamma} r_{1}$ for $m \leq N_{\text {read }}$. We call the region $r_{1}<r<r_{N_{\text {read }}}=N_{\text {read }}^{1 / \gamma} r_{1}$ "semisaturated", since it is unsaturated for some $m<N_{\text {read }}$ reads but saturated in $N_{\text {read }}$ reads. Then for pixels at $r_{m}<r<r_{m+1}$, a total of $\left(r / r_{m}\right)^{-\gamma} n_{\max }$ photoelectrons will be read during $m$ reads, which lies in the range $(m /(m+1), 1) n_{\max }$.

If the source is displaced along the $x$-axis by $\epsilon$ pixels, then this leads to a change in flux $(d \ln F / d \ln r) \epsilon$ compared to a fractional error $[n(r)]^{-1 / 2}$, and hence a signal-to-noise ratio for this one pixel of

$$
\left(\frac{\mathrm{S}}{\mathrm{N}}\right)_{i}^{2}=\left(\frac{\gamma}{r} \epsilon\right)^{2}\left(\frac{r}{r_{m}}\right)^{-\gamma} n_{\max }
$$

Summing over all pixels in the semi-saturated range, taking account of the fact that pixels lying off the $x$ axis contribute to $(\mathrm{S} / \mathrm{N})^{2}$ as $\cos ^{2} \phi$, and turning the sum into an integral, we obtain,

$$
\begin{aligned}
\left(\frac{\mathrm{S}}{\mathrm{N}}\right)_{\mathrm{semi}-\mathrm{sat}}^{2} & =\sum_{i}\left(\frac{\mathrm{S}}{\mathrm{N}}\right)_{i}^{2} \\
& \rightarrow \pi \gamma^{2} \epsilon^{2} n_{\max } \sum_{m=1}^{N_{\text {read }}-1} \int_{r_{m}}^{r_{m+1}} \frac{d r}{r}\left(\frac{r}{r_{m}}\right)^{-\gamma} \\
& =\pi \gamma \epsilon^{2} n_{\max } \sum_{m=2}^{N_{\text {read }}} \frac{1}{m}
\end{aligned}
$$

A similar calculation for the region just beyond the saturation zone gives

$$
\left(\frac{\mathrm{S}}{\mathrm{N}}\right)_{\text {unsat }}^{2}=\pi \gamma \epsilon^{2} n_{\max } .
$$

Hence the error in estimating this offset (e.g., Gould 1995) is

$$
\begin{aligned}
\sigma(\theta) & =\frac{p}{\sqrt{\pi \gamma n_{\max } \ln \left(1.78 N_{\text {read }}+0.9\right)}} \\
& =60 \mu \operatorname{as}\left(\frac{p}{110 \operatorname{mas}}\right)\left(\frac{n_{\text {max }}}{10^{5}}\right)^{-1 / 2} \\
& \times\left(\frac{\ln \left(1.78 N_{\text {read }}+0.9\right)}{3.6}\right)^{-1 / 2}\left(\frac{\gamma}{3}\right)^{-1 / 2}
\end{aligned}
$$

where $p$ is the pixel size $\mathrm{e}^{2}$.

Note that this expression is completely independent of the source brightness: it only requires that the source is saturated. This explains the flat behavior of the astrometric precision in the range $8<H<14$ in Figure 1 . In the range $13<H<16$, the central pixel is unsaturated in a single $2.6 \mathrm{~s}$ read and the semi-saturated region gradually disappears, yielding a gradual transition to standard "root-N" behavior. On the other hand, at brighter magnitudes $H<8$, the diffraction spikes become important and add a new source of "signal". In contrast to the Airy disk, the effective width of the diffraction spikes does not increase with source brightness, so their information content grows directly with source brightness, leading to improved astrometric precision. At the brightest magnitudes $H \lesssim 4$ (which are not of practical interest), the finite $(2048 \times 2048)$ size of the simulated PSF cuts off important regions of astrometric information, which induces a spurious flattening.

\subsection{Photometric Precision of Saturated Broad Band Airy Disk}

A similar calculation for photometric precision yields

$$
\begin{gathered}
\left(\frac{\mathrm{S}}{\mathrm{N}}\right)_{\text {semi-sat,phot }}^{2} \rightarrow 2 \pi n_{\max } \sum_{m=1}^{N_{\text {read }}-1} \int_{r_{m}}^{r_{m+1}} d r r\left(\frac{r}{r_{m}}\right)^{-\gamma} \\
=\frac{2 \pi n_{\max }}{\gamma-2} r_{1}^{2}\left(-N_{\text {read }}^{2 / \gamma}+\sum_{m=1}^{N_{\text {read }}} m^{2 / \gamma-1}\right)
\end{gathered}
$$

which can be expressed

$$
\begin{gathered}
\left(\frac{\mathrm{S}}{\mathrm{N}}\right)_{\text {semi-sat,phot }}^{2}=\pi n_{\text {max }} r_{\text {unsat }}^{2}(1-Q) ; \\
Q \equiv \frac{N_{\text {read }}^{-1}+\gamma\left(2 N_{\text {read }}\right)^{-2 / \gamma}}{\gamma-2},
\end{gathered}
$$

where $r_{\text {unsat }}=N_{\text {read }}^{1 / \gamma} r_{1}$ is the smallest radius at which the full readout is unsaturated. A trivial calculation yields $(\mathrm{S} / \mathrm{N})_{\text {unsat,phot }}^{2}=2 \pi n_{\max }(\gamma-2)^{-1} r_{\text {unsat }}^{2}$. Hence the fractional photometry error is

$$
\begin{aligned}
\sigma(\ln F) & =\left[\pi n_{\max }\left(\frac{\gamma}{\gamma-2}-Q\right)\right]^{-1 / 2} r_{\text {unsat }}^{-1} \\
& =2.1 \times 10^{-4}\left(\frac{n_{\max }}{10^{5}}\right)^{-1 / 2} \\
& \times\left(\frac{\gamma /(\gamma-2)-Q}{2.7}\right)^{-1 / 2}\left(\frac{r_{\text {unsat }}}{5}\right)^{-1}
\end{aligned}
$$

Note that since $r_{\text {unsat }} \propto F^{1 / \gamma}$ the fractional photometry precision scales the same way, i.e., as $F^{1 / 3}$ for a broadband Airy disk. The magenta curve in the middle panel of Figure 1 is a numerical evaluation of the right-hand

\footnotetext{
${ }^{\mathbf{2}}$ We use $\sum_{m=1}^{N} m^{-1} \simeq \ln (1.78 N+0.9)$ rather than the limiting Euler formula because it is accurate for low $N$, down to $N=1$.
} 
side of Equation (16), with $\gamma=3$ and $r_{\text {unsat }}$ evaluated numerically in the top panel from $\pi r_{\text {unsat }}^{2}=\left(N_{\text {sat }}+\right.$ $\left.N_{\text {semi-sat }}\right)$, i.e., the total number of saturated and semisaturated pixels. The agreement is essentially perfect.

Finally, we note that semi-saturated annulus dominates the astrometric information while the unsaturated outer regions dominate the photometric information. However, in neither case is this dominance overwhelming. For the astrometric precision, the ratio (semi-saturated/unsaturated) is 2.6:1, whereas for the photometric precision it is $1: 2.9$. This will have important implications for controlling systematics.

\subsection{Utility of Analytic Formulae}

The fact that the curves in Figure 1 can be understood qualitatively and quantitatively from analytic formulae allows one to quickly estimate the impact of a wide range of changes in system parameters. For example, at this point it is undecided whether WFIRST will have $2.6 \mathrm{~s}$ reads or $5.2 \mathrm{~s}$ reads. If the latter, then $N_{\text {read }} \rightarrow 10$ (rather than 20). Equation (13) then immediately implies a degradation of astrometric precision of $(\ln 36.5 / \ln 18.7)^{1 / 2}=1.11$. The estimate of the change in photometric precision requires one more step. Adopting $\gamma=3$, we find that $Q=0.51$ (rather than $0.31)$. Hence, the photometric precision is degraded by $((3-0.31) /(3-0.51))^{1 / 2}=1.04$.

\section{From Astrometric Measurements to Parallaxes}

Even assuming that there were no errors in single-epoch astrometry other than those due to photon noise, there are still several steps required to go from the ensemble of such measurements to precision parallaxes. We outline these steps as a preliminary to discussing systematic errors.

First, each astrometric measurement is made relative to the pixel grid of the detector, but what is of practical interest is the position of the star relative to a frame of reference set by the sky. Because the observations will be dithered, perhaps over a total range of $20^{\prime \prime}$, the transformation from pixel-grid frame to sky frame must be made for each image. The only sky "object" whose position can be known as well as the very bright stars that are the subject of this paper, is some ensemble of fainter stars that are near-enough (in angle) that their measured pixel separations can be securely translated into angular separations. The choice of this "ensemble" is crucial because in the next step, the absolute parallax of this "object" will have to be measured extremely precisely in order to translate the "relative parallax" described just below (between the "bright star" and the "ensemble object") into an absolute parallax.

With 400,000 (40,000 epochs for each of 10 fields) dithers over $\sim 20^{\prime \prime} \times 20^{\prime \prime}$, the relative offset between pixels (on some definite, but so far unknown scale) will be determined extremely precisely over these separations by the fact that the great majority of these stars are moving relative to each other only according to their relative parallax, proper motion, and, possibly, modeled binary-motion orbits. That is, there will be of order one star per square arcsec, with individual-epoch astrometric precision of $<1 \mu$ as. Hence, from these $\left(4 \times 10^{7}\right) \times\left(4 \times 10^{5}\right) \sim 10^{13}$ measurements, it will be quite straightforward to constrain the relative offset of pixels, including terms for time evolution and for color and well-filling. This will not, by itself, establish an absolute angular scale, a topic to which we return below. However, the fractional error in the derived parallax due to an inaccurate angular scale is simply equal to the fractional error in the scale, which for bulge stars is orders of magnitude below the parallax statistical errors.

The reference ensemble should then be chosen to have similar parallaxes (in $\mu$ as, not distance modulus), but selected primarily on something other than measured parallax in order to minimize bias. An excellent choice would be stars whose color and magnitude are consistent with membership in the bulge red clump (RC) and whose proper motions are consistent with being in the bulge (e.g., with proper motions relative to the mean of bulge stars no greater than $4{\text { mas } y r^{-1}}^{2}$. There will be relatively few foreground contaminants to such a sample, and these can be eliminated using WFIRST relative parallaxes.

Unfortunately, the total number of RC stars within, say $20^{\prime \prime}$ is far too small to measure the mean absolute parallax with the requisite precision. The density of clump stars in the WFIRST microlensing fields is 1$2 \times 10^{5} \mathrm{deg}^{-2}$, meaning that only 1000-2000 lie within this radius. This may sound like a lot, but in these heavily extincted fields, the RC is at Gaia magnitudes of $18 \lesssim G \lesssim 20$ (or fainter in some cases), implying Gaia parallax precisions ${ }^{\mathbf{3}}$ of $100 \mu \mathrm{as} \lesssim \sigma(\pi) \lesssim 300 \mu \mathrm{as}$, and so ensemble precisions of $3 \mu$ as $\lesssim \sigma(\pi) \lesssim 10 \mu$ as, which is impressive but still a factor $15-50$ times larger than the WFIRST individual relative parallax errors for the brightest stars and 3-10 times larger than the $1 \mu$ as (so $\lesssim 1 \%$ ) parallax errors relevant to the precision radius measurements needed for asteroseismology.

However, one could consider the ensemble of all RC stars over the WFIRST fields and fit the difference between local WFIRST relative parallaxes and absolute Gaia parallaxes to a linear (or quadratic) function. In principle, such an approach runs the danger that discrete (so, non-linear) structures in the bulge would systematically corrupt the fit, but such structures (if they existed) would easily show up in the highly precise WFIRST relative parallaxes alone.

\section{Systematic ERrors}

Systematics can potentially degrade and/or undermine the measurement process outlined in Sections 2 and 3 in two distinct ways. First, there can be systematic errors that corrupt the individual measurements, but in a way that is not correlated from measurement to measurement. These effectively add (in quadrature) to

\footnotetext{
$\mathbf{3}^{3}$ http://www.cosmos.esa.int/web/gaia/science-performance
} 
the statistical errors. Hence, they only need to be controlled at the level of the photon noise of individual measurements. For example, for $H=15$ stars (near the RC), the astrometric errors must be controlled at $\lesssim 100 \mu$ as and the photometric errors at $\lesssim 1 \mathrm{mmag}$. On the other hand purely correlated errors must be controlled at levels that are $\sqrt{N}=200$ times smaller, where $N \sim 40,000$ is the number of independent measurements. That is, for $H=15$, at the $0.5 \mu$ as level. Physically, this corresponds to about one Bohr radius on the detector. Even developing an intuition about what can go wrong at this level is challenging.

\subsection{Random Systematics}

We begin with the first issue, uncorrelated systematics. For example, the calculations leading to Figure 1 were made under the assumption that the detector response was uniform across the pixel. This, of course, will not actually be true. However, all that is really required is that the detector response be known as a function of position, not that it be uniform. This position-dependent response will in fact be measured very well. In any given exposure, roughly $10 \%$ of pixels will receive flux from stars at a level larger than the "sky" background, and the flux from these stars, as well as the position of the star will be known almost exactly from the ensemble of 40,000 exposures from each field. Hence, each pixel will be subjected to $400,000 \times 10 \%=40,000$ random flux experiments with stars with a broad range of colors and brightness. The ensemble of these, over $3 \times 10^{8}$ pixels will be used to measure the mean response, so that the 40,000 flux experiments will really only be used to find the deviations from the mean and the time evolution of individual pixels. These same flux experiments will measure pixel response as a function of well-depth and mean wavelength of incident flux. Moreover, as mentioned at the end of Section 2, there will be roughly equal information coming from the semisaturated and unsaturated pixels, which permits internal tests on each individual astrometric or photometric measurement, and which allows to test for extremely subtle deviations from the ensemble of such differences. Similarly, one can search for differences according to angular position of pixels with respect to the center.

Hence, while it is not possible to enumerate in advance all conceivable sources of uncorrelated systematic errors, it will be straightforward to quantify the level of such errors (from excess noise they create) and to construct many possible tests to locate these errors and calibrate them out.

\subsection{Correlated Systematics}

WFIRST microlensing observations will be carried out in six 72-day campaigns, centered on the equinoxes (since the microlensing fields are near the winter solstice). Correlated errors in the parallax measurement will occur if the position of the target star is shifted relative to the ensemble of comparison stars differently in the spring campaigns than the fall campaigns. As emphasized above, effects leading to shifts of one Bohr radius, or even smaller, must be considered.

The two main avenues for such systematics effects are first that the camera is rolled $180^{\circ}$ between equinoxes and second that the Sun is on the opposite side of the field (but the same side of the camera). One can easily imagine that these factors can lead to systematic effects of some sort at the Bohr-radius level. However, it is important to bear in mind that these effects, whatever they are, must operate differently on the target star compared to the ensemble of reference stars. It cannot, for example, have anything to do with the particular pixel that the target star lands on, because it lands on 20,000 different random pixel positions in each of the spring and fall configurations. There are only two properties that are systematically different: brightness and color.

As already mentioned, it is straightforward to test for brightness dependence by comparing the positions (and so parallaxes) derived from the semi-saturated versus unsaturated pixels. Since there are of order $10^{6}$ stars on which this test can be performed, extremely small effects as a function of other control variables (like proximity to the Sun-side of the detector) can be detected. Thus, there are good prospects not only for detecting and measuring such effects, but also determining their origin and thus calibrating them out.

One might think of statistically comparing the mean derived parallax as a function of star brightness, but there is an obvious source of contamination to such a test: brighter stars might be systematically nearer. However, one can compare the mean parallax difference between WFIRST relative parallaxes and Gaia absolute parallaxes as a function of brightness, which is immune to such bias. Similar tests could be done as a function of source color (which is highly correlated with brightness).

Thus while we cannot prove in advance that all such systematics can be controlled, there are at least good prospects for detecting them and, most likely, identifying the source and removing them.

\subsection{Angular Radii}

To determine the physical radius $R$ using a trigonometric-parallax-based distance $d$, one must independently determine the angular radius, $\theta_{*}$, i.e., $R=$ $d \theta_{*}$. This in turn requires knowledge of the star's surface brightness and dereddened flux in the same band. Depending on the precision required, determining these parameters can be non-trivial. The only systematic effort to measure such angular radii from colors and magnitudes in significantly reddened fields of which we are aware is in gravitational microlensing studies, for which the claimed precision (when data are of good quality) is $7 \%$ (e.g., Gould et al. 2014). We first review the established procedure and then outline why one may expect significant improvements for WFIRST targets.

As discussed by Yoo et al. (2004), the mathematical model of the microlensing event yields the instrumental magnitudes of the source (free of blend- 
ing) in several bands, usually including $V$ and $I$. One finds the offset of these values $\Delta((V-I), I)$ from the $\mathrm{RC}$ centroid, using the same instrumental photometry. The dereddened color of the clump is known to be $(V-I)_{0, \mathrm{cl}}=1.06$ from the work of Bensby et al. (2013), which we briefly recount below, while the dereddened magnitude $I_{0, \text { cl }}$ is known as a function of field position from the study by Nataf et al. (2013). These yield $(V-I, I)_{0}=(V-I, I)_{0, \mathrm{cl}}+\Delta((V-I), \Delta I)$. Then this $V / I$ photometry is converted to $V / K$ using the empirical color-color relations of Bessell \& Brett (1988). The $(V-K)_{0}$ color is used to estimate the $K$-band surface brightness employing the empirical color/surfacebrightness relations of Kervella et al. (2004), and finally this is combined with $K_{0}$ to determine the source angular radius, $\theta_{*}$. (See Boyajian et al. 2014, for an update of these relations.)

There are two principal sources of uncertainty in this estimate. First, the dereddened color is determined only to about $\sigma(V-I)_{0} \sim 0.05 \mathrm{mag}$. This uncertainty is known because the color-estimation procedure has been applied by Bensby et al. (2013) to a sample of about 50 dwarfs and subgiants with high-resolution spectra (taken when the source was highly magnified by microlensing). Then the $(V-I)_{0}$ colors were predicted from models based on spectral classification and compared to those determined by the microlensing method. For relatively blue stars near the turnoff, the scatter is about $0.06 \mathrm{mag}$, of which some contribution is due to the uncertainty in the spectroscopic temperature, implying that the intrinsic scatter in the microlensing method is $0.05 \mathrm{mag}$ (or possibly less, if there are other unrecognized errors in the spectroscopic determinations). Redder microlensed stars show greater scatter but Bensby et al. (2013) argue that this is due to uncertainty in the spectroscopic models of these stars. Note that Bensby et al. (2013) determine the color of the RC by choosing the value that minimizes this scatter.

Second, there is typically a $0.1 \mathrm{mag}$ uncertainty in estimating the $I$-band magnitude of the $\mathrm{RC}$ centroid. These two errors combined yield a $7 \%$ error in $\theta_{*}$. No account is usually taken of errors in the overall distance scale (i.e., $R_{0}$ ) derived by Nataf et al. (2013) nor in the color/surface-brightness relations derived by Kervella et al. (2004), since these are deemed small compared to the dominant errors.

The angular radius estimates for giant stars observed by WFIRST will follow the broad outline of microlensing estimates but differ in many particulars. Most important, the main flux measurement will be carried out in the infrared, probably $K$ band, to enable direct comparison with the most stably calibrated color-surface brightness relation. This removes the use of the VIK color-color relations of Bessell \& Brett (1988), which are in fact truly valid only for near-solar metallicity stars.

This will also permit a much longer color baseline for determining the color of RC stars, either in $V-$ $K$ or $I-K$ (for more heavily reddened fields). Note that for these very bright stars, ground-based surveys can provide good $V$ and/or $I$ band photometry, given that WFIRST's own star catalog will permit excellent subtraction of fainter blends in the great majority of cases.

Second, the depth of the RC will be almost completely removed by the relative-parallax measurements coming from WFIRST itself. Of course, the intrinsic "height" of the RC will remain, which has been estimated by Nataf et al. (2013) to be $\sigma\left(M_{I, \mathrm{cl}}\right)=0.09 \mathrm{mag}$, which is 3-4 times smaller than the height dispersion in typical current microlensing fields. This will not only improve the precision of the $\mathrm{RC}$ centroid in the vertical direction, it will permit more restrictive identification of RC stars and (by combining deviations from the centroid in both color and magnitude) more secure identification of RC stars affected by differential extinction. Of course, since WFIRST fields will typically have higher extinction than current microlensing fields, they will also have higher differential extinction at fixed angular scale. This is important because differential extinction induces a difference between the extinction estimate from an ensemble of clump stars in the neighborhood of a given star and the true extinction toward that star. ${ }^{4}$ However, the density of RC stars will also be higher, and since the RC (corrected for distance) is tighter, much smaller angular scales need to be probed to establish the $\mathrm{RC}$ centroid.

Thus, if the color/surface-brightness relations can be well-understood (see discussion below and Gould $2014 \mathrm{~b}$ ), there are good prospects for measuring angular radii to several times better than the $\sim 7 \%$ achieved in current microlensing experiments, i.e., $\sim 2 \%$.

We now turn to the two sources of uncertainty that are presently ignored because they are too small relative to the color and magnitude errors: uncertainty in $R_{0}$ and uncertainty in the color/surface-brightness relations. The first will be all but eliminated by the combination of Gaia and WFIRST. Gaia by itself will determine the mean distance to bulge $\mathrm{RC}$ stars with great precision and WFIRST will tie individual RC stars to this system at extremely high precision.

The second problem is much more severe. The Kervella et al. (2004) color/surface-brightness relations are very tight but the calibration is based on interferometry of stars that are (necessarily) in the solar neighborhood and hence primarily of near-solar metallicity. However, since $V$-band is also sensitive to metallicity through line blanketing, it is far from clear that the empirical relations will apply to some of the extreme stars in the Galactic bulge. To generate a $1 \%$ error in $\theta_{*}$, the $V$ magnitude must be "wrong" (relative to a solarmetallicity star of the same $K$-band surface brightness) by only $0.065 \mathrm{mag}$.

This problem is therefore actually two-fold. First, to be applied to the extremely metal-rich sub-populations that inhabit the Galactic bulge, the relations must be

\footnotetext{
${ }^{4}$ Fortunately, this leads to two effects that mostly cancel. That is, underestimating extinction causes one to underestimate the true source brightness, and so underestimate its radius. However, it also causes one to underestimate the temperature, and so the surface brightness, and hence overestimate the radius.
} 
calibrated as a function of metallicity. Second, if these relations are found to depend on metallicity, then the WFIRST stars to which it is applied must have metallicity measurements (or they will have increased uncertainty due to lack of knowledge of the metallicity).

The problem of calibration is more fundamental. As discussed in detail by Gould (2014b), there are extremely few local calibrators that would be accessible to the workhorse techniques of lunar occultations and long baseline interferometry. He therefore develops a new microlensing-based technique for calibrating the color/surface-brightness relation for metal-rich stars. In essence, for a subset of microlensing events it is possible to measure the source radius crossing time, i.e., $t_{*} \equiv \theta_{*} / \mu$, where $\theta_{*}$ is the source radius and $\mu$ is the lens-source relative proper motion. One then waits a sufficient time $\Delta t$ for the source and lens to have a measurable separation $\Delta \theta$, and then derives $\mu=\Delta \theta / \Delta t$, and so $\theta_{*}=\mu t_{*}$. There are a number of technical challenges to making these measurements, but they do appear to be feasible. We refer the reader to Gould (2014b) for details.

There are approximately 1 million stars with $H<14$ in the WFIRST fields. We show below that this is a conservative boundary for stars with useful astroseismic data. Even with the already existing APOGEE infrared spectrograph, which has 300 fibers, mounted on, e.g., the $2.5 \mathrm{~m}$ DuPont telescope, it would be possible to obtain about $600(\mathrm{~S} / \mathrm{N}=50)$ spectra per night, which is sufficient to determine bulk metallicities. Hence, of order $5 \%$ of the full sample could be measured in a single season, including the great majority of the brighter targets, which can be observed with much shorter exposures. This would be sufficient to determine what additional level of investment was warranted by the asteroseismic data. By the time that WFIRST is launched, more ambitious infrared spectrographs may be in operation. For example, the MOONS ${ }^{\mathbf{5}}$ spectrograph (Oliva et al. 2012) proposed for the $8.2 \mathrm{~m}$ VLT would have 1000 fibers over $500 \operatorname{arcmin}^{2}$. It could obtain $\mathrm{S} / \mathrm{N}=50$ spectra for 1 million targets $H<14$ in a dedicated 40 day campaign.

\section{Simulated WFIRST Asteroseismology}

Our basic approach to simulating WFIRST asteroseismology is to take real Kepler lightcurves and add noise. This implicitly treats Kepler lightcurves as noise-free, which of course is not strictly true, but is appropriate because WFIRST noise is much larger than Kepler noise. Hence, the difference between this and a "correct" approach is much smaller than the uncertainty in estimating the WFIRST noise.

There are two additional adjustments that we must make to the Kepler data beyond adding WFIRST noise. First, we must change the amplitude of the stellar oscillations due to the fact that these are less pronounced in the WFIRST H-band than in the Kepler band. Second, we must account the seasonal gaps in the WFIRST

\footnotetext{
$\mathbf{5}_{\text {http://www.roe.ac.uk/ ciras/MOONS/Overview.html }}$
}

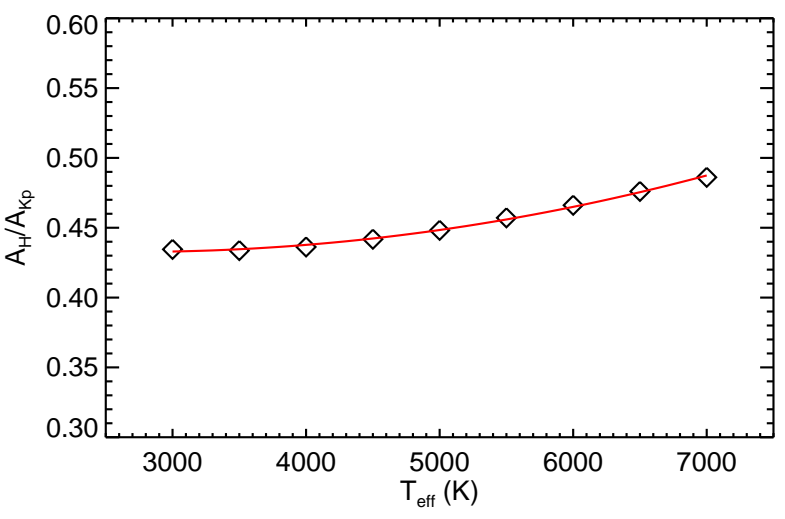

Figure 2. Ratio of $H$-band to $K p$-band amplitude as a function of $T_{\text {eff }}$ assuming blackbody curves and that the luminosity variations are entirely due to changes in temperature. The red solid line shows a second order polynomial fit to the data (Equation (17)).

data that do not occur in Kepler data, since these can introduce aliasing. In addition, we must account for the fact that the WFIRST cadence is twice as fast as the Kepler cadence (15 vs. $30 \mathrm{~min}$ ), but this we do simply by decreasing WFIRST errors by $\sqrt{2}$.

\subsection{Amplitude Adjustment}

We begin by assuming that brightness oscillations are purely due to temperature changes. In principle, radius variation also play a role but these are very small by comparison (Kjeldsen \& Bedding 1995). We approximate the spectral energy distribution by a black body at a given effective temperature $T_{\text {eff }}$ and integrate over the Kepler bandpass

(http://keplergo.arc.nasa.gov/kepler_response_hires1.txt) and the 2MASS $H$-band bandpass respectively. The latter is, of course, not identical to the WFIRST bandpass, but for the reddish stars we are considering, which peak in the WFIRST band and well redward of the central response of the Kepler band, the main sensitivity to temperature is through the Kepler band. Figure 2 shows the resulting ratio of amplitudes $A_{H / K_{p}}$. The main point is that it is essentially flat over a broad range of temperatures. Fitting the points to a parabola, we obtain

$$
A_{H / K_{p}}=0.448+0.068\left(T_{5000}-1\right)+0.074\left(T_{5000}-1\right)^{2}
$$

where $T_{5000} \equiv T_{\text {eff }} / 5000 \mathrm{~K}$. We use Equation (17) in our simulations, although $A_{H} / K p=0.45$ would be a very good approximation.

\subsection{Spectral Frequency Response Function}

The WFIRST microlensing observations will take place in 72-day intervals centered on quadrature. The exact scheduling of the six campaigns has not been decided upon. For simplicity, we approximate that these take place over 3 consecutive years. This schedule then mimics the typical day/night gaps of ground-based observations (except that the cycle is 182 times longer) and 


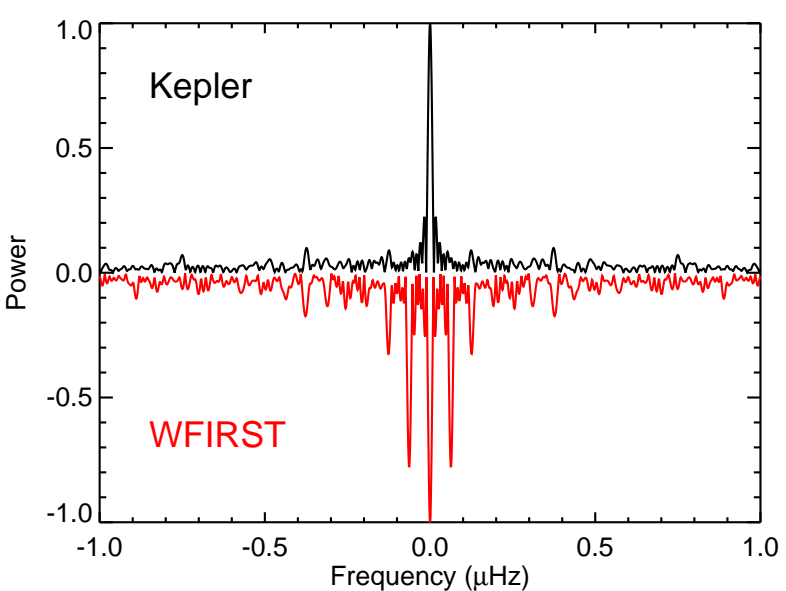

Figure 3. Spectral window function for a typical Kepler time series (top panel, black) and after degrading the time series to a typical duty cycle expected for WFIRST (bottom panel, red). Note that while $\sim 80 \%$ of the power is displaced to side lobes, these are still contained within a $\sim 0.25 \mu \mathrm{Hz}$ envelope, which is substantially narrower than most spectral features of interest.

so can be expected to induce similar aliasing (but with frequency spacings that are 182 times smaller). This is illustrated by Figure 3, where we show a typical Kepler spectral window function and the result of imposing the WFIRST observing windows on the Kepler time series. Note that while $\sim 80 \%$ of the power is now in sidelobes with spacing $\sim 0.06 \mu \mathrm{Hz}$ (corresponding to the 182 day on/off cycle), the entire envelope of this "distributed" power is contained within $\sim 0.25 \mu \mathrm{Hz}$. We will see below that this has no practical impact (except possibly for the very brightest stars) because the frequency spacings that are potentially measurable are much larger than this. Hence, when comparing WFIRST to Kepler, the main difference is photometric noise, to which we now turn.

\subsection{Photometric Noise Estimates}

As discussed in Section 2.2, the photometric noise is a function of apparent brightness, which is in turn determined by three parameters: luminosity, distance, and extinction. Here we focus on stars in the Galactic bulge (distance modulus $\mu=14.7$ ), and adopt a typical extinction for these fields, $A_{H}=0.5$. Therefore, $H=M_{H}+15.2$. We then find from Figure 1 that

$$
\sigma_{W}=1.0 \times 10^{(2 / 15) M_{H}} \mathrm{mmag} .
$$

For each star considered, we evaluate $M_{H}=$ $-2.5 \log \left(L / L_{\odot}\right)+M_{\mathrm{bol}, \odot}-\mathrm{BC}_{H}$ where $M_{\mathrm{bol}, \odot}=4.75$ and $\mathrm{BC}_{H}$ is the $H$-band bolometric correction adopted from Casagrande \& VandenBerg (2014) by interpolating their tables in $T_{\text {eff }}, \log g$, and $[\mathrm{Fe} / \mathrm{H}]$. Then taking account of the fact that WFIRST observations occur twice as frequently as Kepler observations, we create a simulated lightcurve of flux in WFIRST $H$-band, $F_{H, i}$,

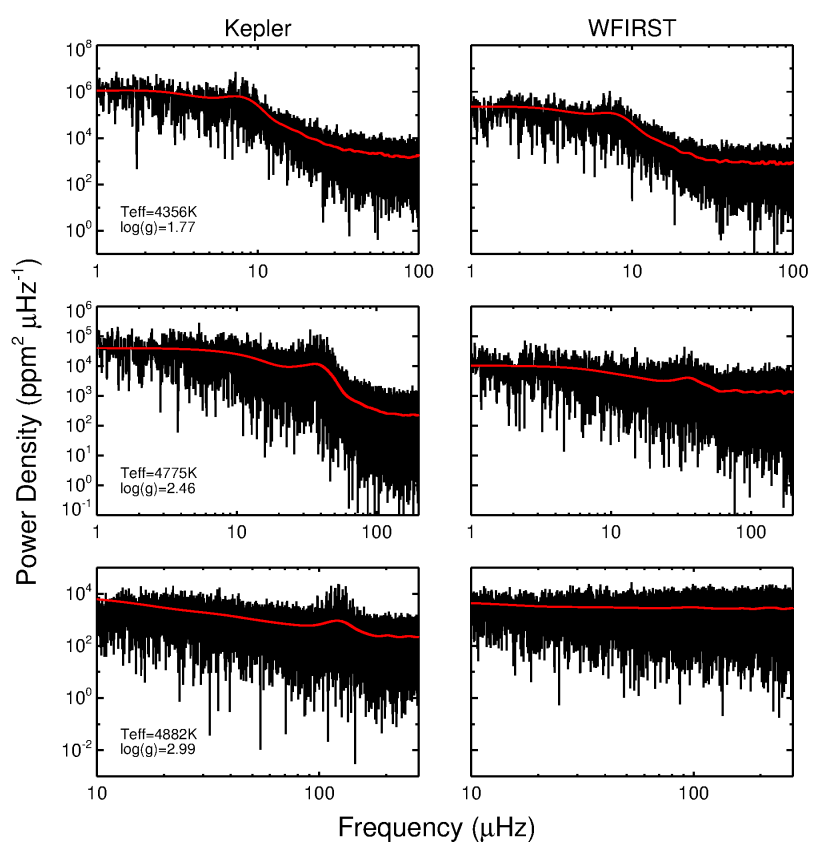

Figure 4. Power spectra of Kepler observations (left panels) and simulated WFIRST observations (right panels) for three red giants in different evolutionary stages: high-luminosity red giant (top panels), red clump (middle panels) and lowluminosity red giant (bottom panels). Red lines show the power spectra smoothed with a Gaussian with a full-width half-maximum of $2 \Delta \nu$. Estimated stellar properties are given in the left panels, with a more complete description given in Table 1.

by

$$
F_{H, i}=\left(F_{K p, i}-\overline{F_{K p}}\right) A_{H / K p}+N\left(0, \frac{\sigma_{W}}{\sqrt{2}}\right),
$$

where $F_{K p, i}$ is the $i$ th observed Kepler flux measurement, $\overline{F_{K p}}$ is the mean of these data points, and $N(q, \sigma)$ is a Gaussian random variable of mean $q$ and variance $\sigma^{2}$.

\subsection{Simulated WFIRST Asteroseimology}

To assess the potential of WFIRST asteroseimology, we simulate $W F I R S T$ observations of three red giants in different evolutionary stages that were observed by Kepler, first "placing them" in the Galactic bulge, behind $A_{H}=0.5 \mathrm{mag}$ of extinction (as described in Section 5.3). These range from the high-luminosity red giant KIC $2437965\left(\nu_{\max } \sim 7.5 \mu \mathrm{Hz}\right)$, through the clump giant KIC $2836038\left(\nu_{\max } \sim 35.7 \mu \mathrm{Hz}\right)$, to the low luminosity red giant KIC $6605620\left(\nu_{\max } \sim 120.5 \mu \mathrm{Hz}\right)$. See Table 1.

Figure 4 shows the power spectra for the original $\mathrm{Ke}$ pler data (left panels) and the simulated WFIRST data (right panels). As expected from the well-known relation between oscillation amplitudes and luminosity (Kjeldsen \& Bedding 1995; Huber et al. 2011), oscillations are more easily detected in high-luminosity giants. Despite the decreased oscillation amplitude and 


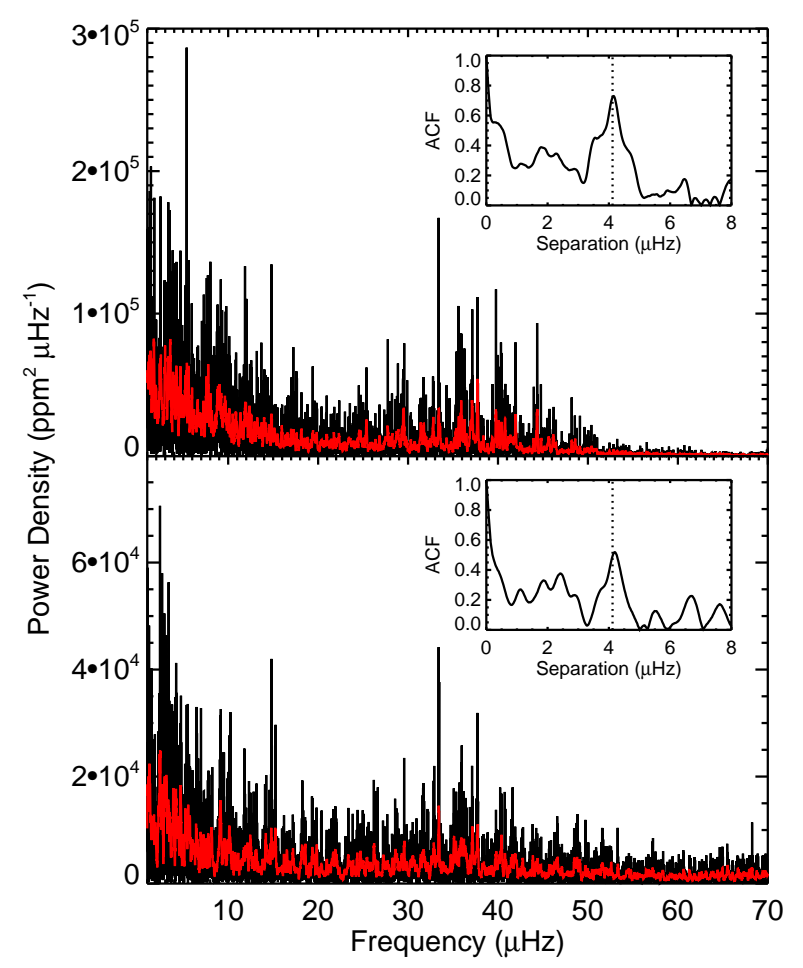

Figure 5. Oscillation spectrum for KIC 2836038 as observed by Kepler (top panel) and as simulated for WFIRST (bottom panel). Red lines are the power spectra smoothed with a boxcar width of $1 \mu \mathrm{Hz}$. The insets show the autocorrelation of the smoothed power spectrum between $20-60 \mu \mathrm{Hz}$ after correcting the granulation background. The dotted line marks the published value for the large frequency separation $\Delta \nu$ (Pinsonneault et al. 2014).

increased photon noise, the oscillations are clearly detectable for the high-luminosity red giant and for the clump giant. For the low-luminosity red giant, both the sloping background granulation and oscillation signals are completely masked by noise. Note that even for the luminous giant, the hump of the oscillation power excess (centered on $\nu_{\max }$ ) has a width that is an order of magnitude larger than the envelope of the spectral window shown in Figure 3. This means that the WFIRST semi-annual window function has no impact on the detectability of this feature.

Figure 5 shows a close-up of the power excess for the red-clump star for Kepler data (top panel) and WFIRST simulated data (bottom panel). The WFIRST power spectrum is clearly affected by the increased noise, which tends to bury individual modes in the noise floor. Note in particular that the Gaussian noise that has been injected into the individual data points results in a "white noise" floor in the Fourier transform, which is absent from the Kepler spectrum for $\nu \gtrsim 55 \mu \mathrm{Hz}$. This will make the extraction of individual frequencies challenging. Importantly, however, the data have sufficiently high $\mathrm{S} / \mathrm{N}$ to allow a clear detection of $\left\langle\Delta \nu_{n l}\right\rangle$. This is demonstrated in the insets, which show an autocorrelation of the spectrum centered
Table 1

Fundamental properties and simulation parameters for WFIRST simulations.

\begin{tabular}{lrrr}
\hline & KIC & KIC & KIC \\
Parameter & 2437965 & 2836038 & 6604620 \\
\hline$T_{\text {eff }}(\mathrm{K})$ & 4356 & 4775 & 4882 \\
$\log g(\mathrm{cgs})$ & 1.765 & 2.460 & 2.993 \\
{$[\mathrm{Fe} / \mathrm{H}](\mathrm{dex})$} & 0.43 & 0.33 & -0.33 \\
$R\left(R_{\odot}\right)$ & 24.94 & 11.28 & 6.50 \\
$M_{H}(\mathrm{mag})$ & -3.14 & -1.60 & -0.45 \\
$A_{H} / A_{K p}$ & 0.44 & 0.45 & 0.45 \\
$\sigma_{W}(\mathrm{mmag})$ & 0.381 & 0.613 & 0.871 \\
Reference & $\mathrm{P} 14$ & $\mathrm{C} 14$ & $\mathrm{C} 14$ \\
\hline
\end{tabular}

References: P14 = Pinsonneault et al. (2014), C14 = Casagrande et al. (2014).

on the power excess. In both cases a clear peak is visible near $4.1 \mu \mathrm{Hz}$, which agrees with the published $\left\langle\Delta \nu_{n l}\right\rangle$ value (Pinsonneault et al. 2014). Because this value is much larger than the $0.06 \mu \mathrm{Hz}$ aliases in the WFIRST spectral window function (induced by semiannual gaps) seen in Figure 4, these aliases do not critically impact measurement of this feature. However, for extremely bright stars with $\sim 10$ times larger radii $(\sim 1000$ times lower density, and so $\sim 30$ times smaller $\left\langle\nu_{n l}\right\rangle$ ), this would become an issue (assuming that their $\sim 1$ yr periods were adequately resolved in the WFIRST mission). Note that the autocorrelations were calculated using power spectra that were corrected for the granulation background as described by Huber et al. (2009). While the exact detection limit will depend on the actual photometric performance, these tests confirm that WFIRST has great potential for asteroseismology of red giants above and slightly below the red clump.

In evaluating this potential, it is important to keep in mind that WFIRST astrometry will help to distinguish genuine seismic detections from instrumental power excess (e.g., Figure 6 from Hekker et al. 2011), as well as noise spikes and aliases. For example, luminosities based on WFIRST parallaxes will provide a good initial guess for the location of the power excess due to oscillations (Stello et al. 2008). Furthermore, WFIRST parallaxes (Section 2.1) combined with flux and surfacebrightness measurements (Section 4.3) will yield the radius $R$, and as outlined in Section 1, this can be combined with the measurement of $\nu_{\max }$ (from the middle panel of Figure 5) to estimate the mass, even without a direct measurement of $\left\langle\nu_{n l}\right\rangle$ (Equation (5)). But this also means that the approximate location of $\left\langle\nu_{n l}\right\rangle$ can be determined from the measurement $\nu_{\max }$ and $R$, via

$$
\frac{\left\langle\Delta \nu_{n l}\right\rangle}{\left\langle\Delta \nu_{n l}\right\rangle_{\odot}} \simeq\left(\frac{\nu_{\max }}{\nu_{\max , \odot}}\right)^{1 / 2}\left(\frac{T_{\mathrm{eff}}}{T_{\mathrm{eff}, \odot}}\right)^{1 / 4}\left(\frac{R}{R_{\odot}}\right)^{-1 / 2}
$$

Then, once the location of this peak is approximately identified from the measurements of $\nu_{\max }$ and $R$ (and these scaling relations), it can be measured more precisely from the autocorrelation function. A simpler version of this procedure is already in routine use (e.g., 


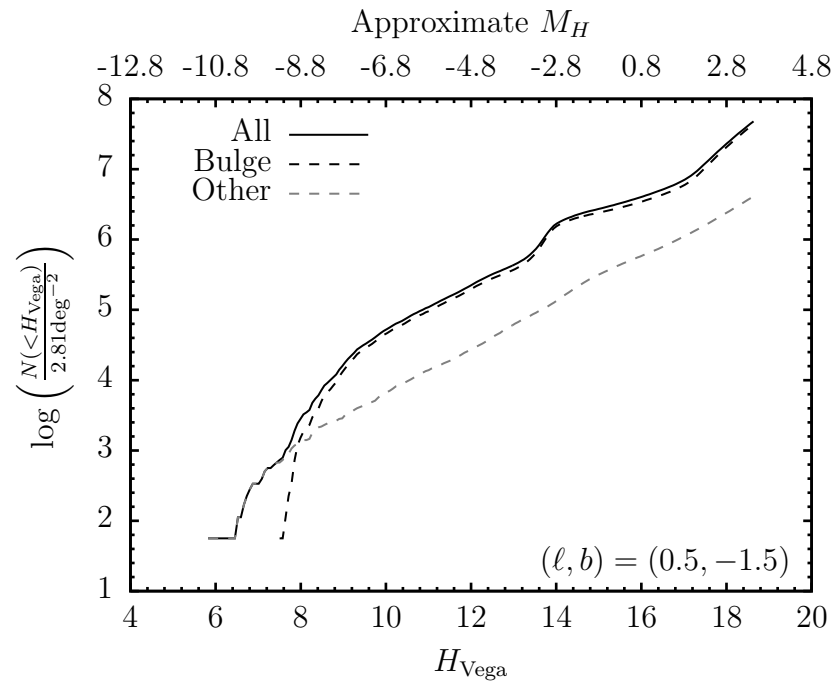

Figure 6. Cumulative distribution of stars in a typical choice for the $2.8 \mathrm{deg}^{2}$ WFIRST field toward the Galactic bulge. Solid curve represents all stars while dashed curve represents stars lying in the Galactic bulge. To understand anticipated astroseismic performance of WFIRST, one should subtract $\mu+A_{H}=15.2$ from the observed $H$ magnitude and compare with the absolute magnitudes $M_{H}$ shown in Table 1 as displayed in Figure 4. This is strictly true for bulge stars and approximately true for the others, which are mainly in the foreground disk. WFIRST will return very good astroseismic data for about 1 million stars. Upper scale shows approximate absolute magnitude, assuming Galactocentric distance and fiducial extinction.

Huber et al. 2009) based on the purely asteroseismic empirical scaling relation of Stello et al. (2009),

$$
\frac{\left\langle\Delta \nu_{n l}\right\rangle}{\mu \mathrm{Hz}}=(0.263 \pm 0.009)\left(\frac{\nu_{\max }}{\mu \mathrm{Hz}}\right)^{0.772 \pm 0.005} .
$$

However, the additional radius information entering Equation (20) makes it more robust against unusual stars that systematically differ from the local calibration sample underlying Equation (21). Such stars may be more common in the Galactic bulge than they are locally. If the autocorrelation function is too noisy to identify a clear peak even with this assist, or if the noise renders the error in the position of the peak too large to be usable. it will still be possible to estimate the mass, just from the measurements of $\nu_{\max }$ and $R$ via Equation (5).

\subsection{Availability of Asteroseismic Targets}

Figure 6 shows the cumulative distribution of stars by $H$-band magnitude for a typical choice of the $2.8 \mathrm{deg}^{2}$ WFIRST field, estimated using the TRILEGAL Galactic model (Vanhollebeke et al. 2009; Girardi et al. 2012). The subset of these that lie in the Galactic bulge are delineated by the bold dashed line. The bump near $H \sim 13.5$ is the red clump. The quality of the asteroseismic data for the bulge stars can be directly evaluated by comparison to the three stars shown in Figure 4 , whose parameters are given in Table 1 . These have $M_{H}=(-3.1,-1.6,-0.5)$, so in our approximation of uniform extinction, $H=(12.1,13.6,14.7)$. The remaining stars are overwhelmingly in the foreground Galactic disk and so are dimmer at fixed apparent magnitude. Thus, at the same $H$ magnitude they will, of course, have the same photometric errors, but being dimmer, their asteroseismic signals will generally be somewhat weaker. Nevertheless, because most of these stars lie within a magnitude of the distance modulus of the bulge, Figure 4 provides a good qualitative indicator of astroseismic performance for these stars as well as a function of magnitude.

Thus, in total, there will be roughly 1 million stars with $H<14$ with good astroseismic solutions, particularly when account is taken of the precision parallaxes (and so radii) for these stars from WFIRST astrometry. In particular, for $H=14$, these will have $\sigma(\pi)=0.34 \mu$ as parallaxes, corresponding to $0.3 \%$ distance errors.

\section{ACKNOWLEDGMENTS}

Work by AG was supported by NSF grant AST 1103471 and NASA grant NNX12AB99G. MP acknowledges support by The Thomas Jefferson Chair for Discovery and Space Exploration. DH acknowledges support by NASA Grant NNX14AB92G issued through the Kepler Participating Scientist Program.

\section{REFERENCES}

Belkacem, K., Goupil, M. J., Dupret, M. A., et al. 2011, The Underlying Physical Meaning of the $\nu_{\max }-\nu_{\mathrm{c}}$ Relation, A\&A, 530, A142

Bensby, T., Yee, J. C., Feltzing, S., et al. 2013, Chemical Evolution of the Galactic Bulge as Traced by Microlensed Dwarf and Subgiant Stars. V. Evidence for a Wide Age Distribution and a Complex MDF, A\&A, 549, A147

Bessell, M. S., \& Brett, J. M. 1988, JHKLM Photometry - Standard Systems, Passbands, and Intrinsic Colors, PASP, 100, 1134

Boyajian, T. S., van Belle, G., \& von Braun, K. 2014, Stellar Diameters and Temperatures. IV. Predicting Stellar Angular Diameters, AJ, 147, 47

Brown, T. M., Gilliland, R. L., Noyes, R. W., \& Ramsey, L. W. 1991, Detection of Possible P-Mode Oscillations on Procyon, ApJ, 368, 599

Casagrande, L., \& VandenBerg, D. A. 2014, Synthetic Stellar Photometry - I. General Considerations and New Transformations for Broad-Band Systems, MNRAS, 444, 392

Casagrande, L., Silva, A. V., Stello, D., et al. 2014, Strmgren Survey for Asteroseismology and Galactic Archaeology: Let the SAGA Begin, ApJ, 787, 110

Chaplin, W. J., \& Miglio, A. 2013, Asteroseismology of Solar-Type and Red-Giant Stars, ARA\&A, 51, 353

Epstein, C. R., Elsworth, Y. P., Johnson, J. A., et al. 2014, Testing the Asteroseismic Mass Scale Using Metal-Poor Stars Characterized with APOGEE and Kepler, ApJ, 785, L28

Frandsen, S., Lehmann, H., Hekker, S., et al. 2013, KIC 8410637: a 408-Day Period Eclipsing Binary Containing a Pulsating Giant Star, A\&A, 556, A138 
Girardi, L., Barbieri, M., Groenewegen, M. A. T., et al. 2012, TRILEGAL, a TRIdimensional modeL of thE GALaxy: Status and Future, in Red Giants as Probes of the Structure and Evolution of the Milky Way (Berlin: Springer-Verlag), 165

Gould, A. 1995, Analytic Error Estimates, ApJ, 440, 510

Gould, A. 2014a, WFIRST Ultra-Precise Astrometry I: Kuiper Belt Objects, JKAS, 47, 279

Gould, A. 2014b, A New Method to Calibrate the Stellar Color/Surface-Brightness Relation, JKAS, 47, 153

Gould, A., Udalski, A., Shin, J.-G., et al. 2014, A Terrestrial Planet in $\mathrm{a} \sim \mathrm{AU}$ Orbit Around One Member of $\mathrm{a} \sim 15$ AU Binary, Science, 345, 46

Hekker, S., Elsworth, Y., De Ridder, J., et al. 2011, SolarLike Oscillations in Red Giants Observed with Kepler: Comparison of Global Oscillation Parameters from Different Methods, A\&A, 525, A131

Huber, D., Stello, D., Bedding, T. R., et al. 2009, Automated Extraction of Oscillation Parameters for Kepler Observations of Solar-Type Stars, Communications in Asteroseismology, 160, 74

Huber, D., Bedding, T. R., Stello, D., et al. 2011, Testing Scaling Relations for Solar-Like Oscillations from the Main Sequence to Red Giants Using Kepler Data, ApJ, 743,143

Huber, D., Ireland, M. J., Bedding, T. R., et al. 2012, Fundamental Properties of Stars Using Asteroseismology from Kepler and CoRoT and Interferometry from the CHARA Array, ApJ, 760, 32

Kallinger, T., Weiss, W. W., Barban, C., et al. 2010, Oscillating Red Giants in the CoRoT Exofield: Asteroseismic Mass and Radius Determination, A\&A, 509, A77

Kervella, P., Thévenin, F., Di Folco, E., \& Ségransan, D. 2004, The Angular Sizes of Dwarf Stars and Subgiants. Surface Brightness Relations Calibrated by Interferometry, A\&A, 426, 297

Kjeldsen, H., \& Bedding, T. R. 1995, Amplitudes of Stellar Oscillations: the Implications for Asteroseismology, A\&A, 293, 87

Koch, D. G., Borucki, W. J., Basri, G., et al. 2010, Kepler Mission Design, Realized Photometric Performance, and
Early Science, ApJ, 713, L79

Miglio, A., Chiappini, C., Morel, T., et al. 2013, Differential Population Studies Using Asteroseismology: Solar-Like Oscillating Giants in CoRoT Fields LRc01 and LRa01, EPJWC, 4303004

Nataf, D. M., Gould, A., Fouqué, P., et al. 2013, Reddening and Extinction toward the Galactic Bulge from OGLEIII: The Inner Milky Way's RV $\sim 2.5$ Extinction Curve, ApJ, 769, 88

Oliva, E., Diolaiti, E., Garilli, B., et al. 2012, The Design of the MOONS-VLT Spectrometer, SPIE, 8446V

Pinsonneault, M. P., Elsworth, Y., Epstein, C., et al. 2014, The APOKASC Catalog: An Asteroseismic and Spectroscopic Joint Survey of Targets in the Kepler Fields, ApJS, 215, 19

Sandquist, E., Mathieu, R. D., Brogaard, K., et al. 2013, A Long-Period Totally Eclipsing Binary Star at the Turnoff of the Open Cluster NGC 6819 Discovered with Kepler, ApJ, 762, 58

Stello, D., Bruntt, H., Preston, H., \& Buzasi, D. 2008, Oscillating K Giants with the WIRE Satellite: Determination of Their Asteroseismic Masses, ApJ, 674, L53

Stello, D., Chalin, W. J., Basu, S., Elsworth, Y., \& Bedding, T. R. 2009, The Relation between $\Delta \nu$ and $\nu_{\max }$ for SolarLike Oscillations, MNRAS, 400, L80

Ulrich, R. K. 1986, Determination of Stellar Ages from Asteroseismology, ApJ, 306, 37

Vanhollebeke, E., Groenewegen, M. A. T., \& Girardi, L. 2009, Stellar Populations in the Galactic Bulge. Modelling the Galactic Bulge with TRILEGAL, A\&A, 498, 95

White, T. R., Bedding, T. R., Stello, D., et al. 2011, Calculating Asteroseismic Diagrams for Solar-Like Oscillations, ApJ, 743, 161

White, T. R., Huber, D., Maestro, V., et al. 2013, Interferometric Radii of Bright Kepler Stars with the CHARA Array: Cygni and 16 Cygni A and B, MNRAS, 433, 1262

Yoo, J., DePoy, D. L., Gal-Yam, A., et al. 2004, OGLE2003-BLG-262: Finite-Source Effects from a Point-Mass Lens, ApJ, 603, 139 